\title{
EXAFS Study of the Local Structure of Bismuth Film Deposited at Liquid Nitrogen Temperature
}

\author{
Hiroyuki Ikemoto* and Taku Watanabe \\ Department of Physics, University of Toyama, Toyama 930-8555, Japan
}

Takafumi Miyanaga

Department of Advanced Physics, Hirosaki University, Hirosaki 036-8561, Japan

(Received 13 September 2013; Accepted 8 October 2013; Published 19 October 2013)

\begin{abstract}
The local structure of bismuth film deposited at liquid nitrogen temperature was investigated by the extended X-ray absorption fine structure (EXAFS) analysis. In the Fourier transform of EXAFS function the peak originated from the intralayer first nearest neighbor $(1 \mathrm{NN})$ correlation exists while that originated from the interlayer $1 \mathrm{NN}$ correlation disappears. This suggests that the primary structure remains but the secondary structure is disrupted. [DOI: 10.1380/ejssnt.2013.110]
\end{abstract}

Keywords: Bismuth; Amorphous thin films; EXAFS

\section{INTRODUCTION}

Bismuth has a rhombohedral crystal structure, with a lattice parameter $\mathrm{a}=4.5460 \AA$ and $\alpha=57.23^{\circ}$ at $298 \mathrm{~K}$ [1]. In bismuth crystal (c-Bi) Bi atoms are covalently bonded with three nearest neighbor atoms and form bilayers, which stack along the trigonal [001] axis. The atoms make weak bonds in the backward direction with the three neighbors of the adjacent layer. The interlayer interactions cause semimetallic behavior [2]. So the bismuth is characterized by having a hierarchic structure.

The hierarchic structure affects the variation of the structural parameters of c-Bi. When the temperature decreases from 298 to $78 \mathrm{~K}$, a-axis shrinks in $0.25 \%$ while c-axis shrinks in $0.41 \%$ [1]. The anisotropic temperature variation appears also in the atomic distances, that is, the first nearest neighbor atomic distance for the intralayer shortens in $0.23 \%$ while that for the interlayer shortens in $0.37 \%$ [3]. These differences are due to the characters of the hierarchic structure, that is, the strong (the intralayer) and the weak (the interlayer) interactions show different temperature variations.

Bismuth films deposited at liquid helium (LHe) temperature are amorphous superconductor. Observations of electron-diffraction-patterns show that 20 30-nm-thick films deposited onto a collodion film at $4.2 \mathrm{~K}$ are amorphous but crystallize around $20 \mathrm{~K}$ [4]. The crystallization temperature decreases with increasing film thickness [5].

It is interesting to know what kind of structure an amorphous bismuth (a-Bi) takes in the point of view of the hierarchic structure. Extended X-ray absorption fine structure (EXAFS) is the strong tool for comparing the local structures between the crystal and the amorphous materials. It provides the comparable quality of the structural information in both contrary to the X-ray diffraction measurements. Due to the technical difficulties and the layout of the EXAFS beam line, we did the sample preparation and the EXAFS measurements at liquid nitrogen $\left(\mathrm{LN}_{2}\right)$ temperature instead of LHe temperature. Despite of the experiment at $\mathrm{LN}_{2}$ the structure of the prepared $\mathrm{Bi}$ is clearly different from that for $\mathrm{c}-\mathrm{Bi}$.

*Electronic address: ikemoto@sci.u-toyama.ac.jp
We present the local structure of bismuth film deposited at $\mathrm{LN}_{2}$ temperature as the beachhead for investigating the local structure of a-Bi.

\section{EXPERIMENTAL}

In advance of deposition of $\mathrm{Bi} \mathrm{NaCl}$ of $99.99 \%$ purity was deposited on a carbon sheet at room temperature. $\mathrm{Bi}$ of $99.999 \%$ purity was deposited onto $\mathrm{NaCl}$ at $\mathrm{LN}_{2}$ temperature. The thickness of Bi film was $300 \mathrm{~nm}$, which was monitored with a quartz oscillator system (ULVAC, CRTM6000 and CRTS-4). After the deposition of Bi the XAFS measurements were performed with maintaining the sample temperature at $\mathrm{LN}_{2}$ temperature. In order to the investigate annealing effect of the sample the another XAFS measurement was done after the short annealing ( 1 hour) at the room temperature. The as-deposited Bi and the annealed $\mathrm{Bi}$ film are abbreviated to as-depo $\mathrm{Bi}$ and annealed $\mathrm{Bi}$, respectively.

$\mathrm{X}$-ray absorption measurements were performed using the spectrometer installed at BL12C of the Photon Factory $(\mathrm{PF})$ in the High Energy Accelerator Research Organization (KEK), Tsukuba, Japan. EXAFS spectra were measured for Bi $\mathrm{L}_{\mathrm{III}}$-edge $(13.404 \mathrm{keV})$ by the transmission mode. The intensities of incident beam and the transmitted beam were monitored by ionization chambers. We set up the sample in the grazing incidence setup to extend the path length of the transmission X-ray. EXAFS data was analyzed by our program of miXAFS code [8]. Phase shift, backscattering amplitude and electron mean free path were calculated by the FEFF8 code [6].

\section{RESULTS}

Figure 1 displays the EXAFS oscillations $k^{2} \chi(k)$ of the as-depo $\mathrm{Bi}$ and the annealed $\mathrm{Bi}$ as a function of $k . k^{2} \chi(k)$ of the Bi film deposited at room temperature is shown also in Fig. 1 for comparison. All EXAFS data were measured at $\mathrm{LN}_{2}$ temperature. The film deposited at room temperate is confirmed to be crystal, so the film is abbreviated to c-Bi. Distinct EXAFS oscillations are observed up to $18.0 \AA^{-1}$. The period of oscillation for the as-depo 


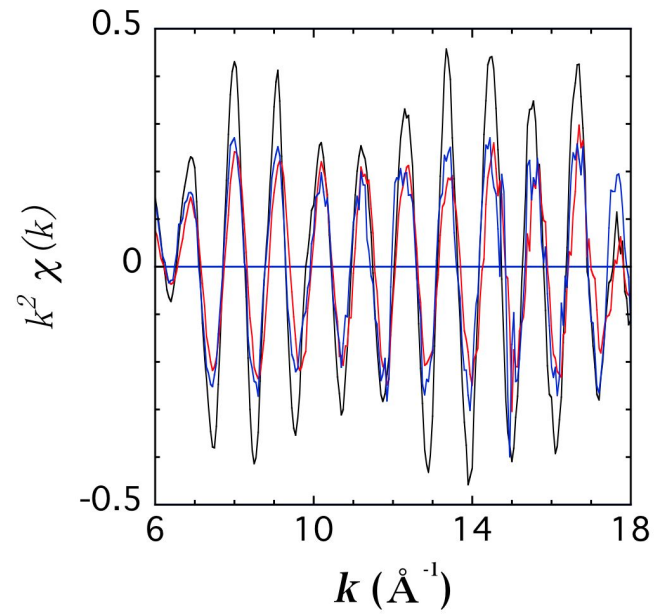

FIG. 1: EXAFS $k^{2} \chi(k)$ spectra for as-depo Bi (red solid line), annealed Bi (blue solid line), and c-Bi (black solid line).

$\mathrm{Bi}$ and the annealed $\mathrm{Bi}$ are almost same as that for $\mathrm{c}-\mathrm{Bi}$, but thier amplitudes are about two thirds of that of $\mathrm{c}-\mathrm{Bi}$.

The Fourier transform (FT) of the $k \chi(k)$ data provides useful informations for identifying atomic correlations. $k \chi(k)$ was Fourier transformed in the $k$-range from 6.0 to $18.0 \AA^{-1}$, where the Hamming window was used to reduce the ripples in the Fourier-transformed spectra in $r$-space. Figure 2 shows the FT of $k \chi(k)$ as a function of the radial distance. There are four prominent peaks at 3.0, 3.6, 4.5 and $5.6 \AA$ in FT of c-Bi. By comparing these peaks with the XRDs in the literature [3], we can assign the first and second peaks to contributions from the intralayer first nearest neighbor $(1 \mathrm{NN})$ and the interlayer $1 \mathrm{NN}$ distances, respectively. The third peak corresponds to the second nearest neighbor distances of the intra- and inter-layer, i.e., 4.55 and $4.75 \AA$, respectively. The fourth peak corresponds to the third nearest neighbor distances of the intra- and inter-layer, i.e., 5.51 and $5.62 \AA$, respectively.

In the FT of as-depo Bi the intensity is two thirds of that for $\mathrm{c}-\mathrm{Bi}$, but the higher-order peaks even including of the second peak are diminished. These results indicate that in as-depo Bi the layer structures are preserved, while the interlayer interactions are diminished.

To extract the peaks originating from the intralayer $1 \mathrm{NN}$, the FT peaks were filtered in the corresponding area. For detailed study of the intralayer $1 \mathrm{NN}$, the least-squares curve-fitting calculations were applied to the Fourier-filtered $\chi(k)$. Table I shows the values of the atomic distance $(r)$, the coordination number $(N)$, the Debye-Waller factor $(D W)$, and the third cummulant $\left(C_{3}\right)$ for the intralayer $1 \mathrm{NN}$. The scaling factor was adjusted by the value of $N$ for c-Bi to become three, which is corresponding to the coordination number of c-Bi. Table I also contains the value of $r$ obtained by X-ray diffraction measurements [3].

Our value of $r$ from EXAFS for c-Bi is same as that of the literature. The value of $r$ for as-depo $\mathrm{Bi}$ is shorter in $0.023 \AA$ than that for $\mathrm{c}-\mathrm{Bi}$, but the value recovers to that for $\mathrm{c}-\mathrm{Bi}$ by annealing at room temperaure. The value of $N$ for the as-depo $\mathrm{Bi}$ and the annealed $\mathrm{Bi}$ are about two

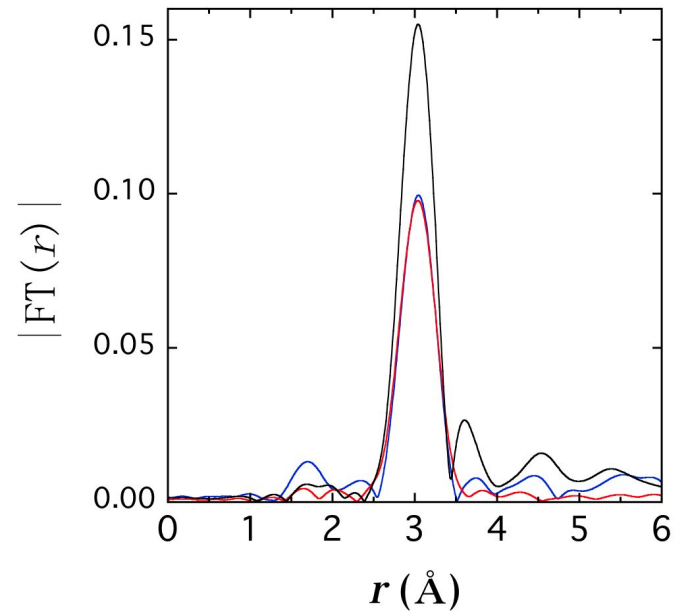

FIG. 2: Fourier transform magnitude of EXAFS $k \chi(k)$ data for as-depo Bi (red solid line), annealed Bi (blue solid line), and $\mathrm{c}-\mathrm{Bi}$ (black solid line).

TABLE I: Structure parameters atomic distance $(r)$, coordination number $(N)$, and Debye Waller factor $(D W)$.

\begin{tabular}{cllll}
\hline \hline & $r(\AA)$ & $N$ & $D W[\AA]$ & $C_{3} \times 10^{-5}\left[\AA^{3}\right]$ \\
\hline c-Bi & 3.064 & 3.00 & 0.0557 & 2.7 \\
as-depo Bi & 3.041 & 2.24 & 0.0622 & -0.3 \\
annealed Bi & 3.066 & 2.05 & 0.0574 & 5.9 \\
c-Bi [3] & 3.064 & 3.00 & & \\
\hline \hline
\end{tabular}

thirds of that for c-Bi. $D W$ for the as-depo $\mathrm{Bi}$ is larger in $20 \%$ than those for $\mathrm{c}-\mathrm{Bi}$ and the annealed $\mathrm{Bi}$. The increase of the value for $D W$ means the increase of the structural disorder or thermal fluctuations. There is a strong correlation between $N$ and the $D W$, so it is not easy to show the conclusive values of them. Then we will withhold the discussion for $N$ and $D W$ in this article.

\section{DISCUSSION}

The electron diffraction measurements show that the thin Bi films deposited at low temperature are amorphous, but they crystallize with slight increase of the temperature [4]. The crystallization temperature decrease with increasing of the Bi film thickness [5]. The powder X-ray diffraction measurements show that the crystal structure of the crystal $\mathrm{Bi}$ does not change in the temperature range between 4 and $300 \mathrm{~K}[3]$.

Despite of the insufficient low temperature for making the a-Bi, FT of the as-depo Bi implies that the interlayer interactions diminish and the intralayer $1 \mathrm{NN}$ atomic distance is slightly shorter than that for c-Bi. This suggests that the as-depo $\mathrm{Bi}$ is amorphous or at least different from the rhombohedral $\mathrm{Bi}$. The electron diffraction measurements [4] reported the appearance of the Bragg peaks with the slight increase of temperature, but they didn't provide any data for the crystal. It is important to know what kinds of Bragg peaks appear, especially the Bragg peaks parallel to $\mathrm{Bi}$ (001). One possible reason for the 
discrepancy in the crystallization temperature is the difference of the substrates. $\mathrm{NaCl}$ was used as the substrate in our experiment, while the collodion film or the sapphire single crystal were used in the electron diffraction measurements $[4,5]$.

Due to the lack of the data of the diffraction measurements, it is impossible to discuss the medium and the long range orders for the $\mathrm{Bi}$ film deposited at $\mathrm{LN}_{2}$ temperature at the present stage. So we will focus on the only local structure of the sample following discussion. EXAFS analysis provides two important results for the sample. One is the disappearance of the interlayer correlation and the other is shrinkage of the covalent bond length.

Tellurium is also characterized by having the hierarchic structure like bismuth. In the trigonal tellurium the reduction of the interlayer distance by the compression induces the slight elongation of the covalent bond length [7]. In the tellurium nanoparticles the reduction of the interchain coordination number brings about the shortening of the covalent bond [8]. In the tellurium the secondary structure (the interchain interactions) affect the primary structure (the covalent bond length). In the materials of the hierarchic elements there are differences in the atomic interactions according to the order of the hierarchy. The higher order structure have generally the weaker interactions.

The anologous phenomena occurs in the bismuth. The primary structure (the intralayer structure) is formed by the covalent bonds, and the secondary structure (the interlayer structure) is constructed by the overlapping between the antibonding orbitals on adjacent layers. The interaction of the secondary structure is weaker than that of the primary structure, so the secondary structure disappears while the primary structure remains in the as-depo Bi. There is a possibility that the secondary structure (the interlayer interactions) changes the primary structure (the covalent bond length) also in the bismuth like the tellurium. The disappearance of the interlayer correlation may cause the shortening of the covalent bond length.

\section{CONCLUSION}

We performed EXAFS analysis of Bi film deposited and measured at $\mathrm{LN}_{2}$ temperature. In the Bi film deposited at $\mathrm{LN}_{2}$ temperature the intralayer correlation is preserved while the interlayer one is diminished. The covalent bond length shortens compared to that of $\mathrm{c}-\mathrm{Bi}$ as observed in Te nanoparticles.

\section{Acknowledgments}

The authors thank Mr. H. Maekawa, R. Imai, and A. Oono and Ms.M. Seo for their assistance at various stages. This work was supported by JSPS KAKENHI Grant Number 23510120 and the Kurata Memorial Hitachi Science and Technology Foundation. The synchrotron radiation experiments were performed at the Photon Factory in KEK under Proposal No. 2008G509, and 2010G612.
[1] D. Schiferl and C. S. Barrett, J. Appl. Cryst. 2, 30 (1969).

[2] N. F. Mott and E.A. Davis, Electronic Progress in NonCrystalline Materials, 2nd Ed. (Clarendon Press, Oxford, 1979), p. 413.

[3] P. Cucka and C. S. Barrett, Acta Cryst. 15, 865 (1962).

[4] S. Fujime, Jpn. J. Appl. Phys. 5, 764 (1966).

[5] B. I. Belevtsev, Y. F. Komnik, and L. A. Yatsuk, Sov. Phys.-JETP, 38, 1255 (1974).
[6] A. L. Ankudinov, B. Ravel, J. J. Rehr, and S. D. Conradson, Phys. Rev. B 58, 7565 (1998).

[7] R. Keller, W. B. Holzapfel, and H. Schulz, Phys. Rev. B 16, 4404 (1977).

[8] H. Ikemoto, A. Goyou, and T. Miyanaga, J. Phys. Chem. C 115, 2931 (2011). 